\title{
Precision measurement of gravity with cold atoms in an optical lattice and comparison with a classical gravimeter.
}

\author{
N. Poli, F.-Y. Wang* M. G. Tarallo, A. Alberti丹 M. Prevedelli $丹$ and G. M. Tind \\ Dipartimento di Fisica e Astronomia and LENS, Università di Firenze \\ INFN Sezione di Firenze, Via Sansone 1, 50019 Sesto Fiorentino, Italy
}

(Dated: October 26, 2018)

\begin{abstract}
We report on a high precision measurement of gravitational acceleration using ultracold strontium atoms trapped in a vertical optical lattice. Using amplitude modulation of the lattice intensity, an uncertainty $\Delta g / g \approx 10^{-7}$ was reached by measuring at the $5^{\text {th }}$ harmonic of the Bloch oscillation frequency. After a careful analysis of systematic effects, the value obtained with this microscopic quantum system is consistent with the one we measured with a classical absolute gravimeter at the same location. This result is of relevance for the recent interpretation of related experiments as tests of gravitational redshift and opens the way to new tests of gravity at micrometer scale.
\end{abstract}

PACS numbers: 91.10.Pp, 03.75.Dg, 37.25.+k, 37.10.Jk

Atom interferometry, and in general methods based on quantum interference of ultracold atoms, were largely used in recent years for gravitational physics experiments and new exciting prospects can be envisioned in the near future [1]. For example, Raman interferometry was used for precise measurements of Earth's gravitational acceleration $g$ 2] and its gradient [3], for determining the value of the gravitational constant [4, 5], for a possible redefinition of the $\mathrm{kg}[6]$, and for geophysical applications [7]. Schemes based on Bloch oscillations of atoms trapped in vertical optical lattices were also used to measure gravity with the possibility of combining high sensitivity and micrometric spatial resolution [8-10]. The results of atom interferometry experiments were interpreted as tests of the isotropy of post-Newtonian gravity [11], of quantum gravity [12], and of gravitational redshift [13]. Prospects include high precision tests of the weak equivalence principle [14, 15], the detection of gravitational waves [16, 17], and future experiments in space [18].

So far, however, Bloch oscillation measurements had limited accuracy compared to Raman atom interferometers. Here, we present a precision measurement of gravitational acceleration $g$ with a new method based on ultracold ${ }^{88} \mathrm{Sr}$ atoms trapped in an amplitude-modulated vertical optical lattice [19] and compare the result with the value obtained with a classical absolute gravimeter based on a Michelson interferometer with one arm including a freely-falling corner-cube. We also improved our previous observation of long-lived Bloch oscillations [9] and discuss the precision of the two methods for the determination of $g$. In addition to demonstrating the sensitivity and accuracy of this new method, our data can be interpreted as a measurement of the gravitational redshift to the Compton frequency of $\mathrm{Sr}$ matter waves, as suggested by H. Müller et al. [13]. Our data surpasses previous Bloch oscillation measurements by one order of magnitude, making it the most precise test of the gravitational redshift based on Bloch oscillations at micrometric spatial scales. The interpretation of atom interferometer redshift tests is complicated by special relativistic time dilation since the atoms are moving [20, 21], but Bloch oscillations experiments with stationary lattices provide a measurement of the purely gravitational effect.

The experimental setup is based on cooled and trapped ${ }^{88} \mathrm{Sr}$ atoms [9] (Fig. 1). Atoms from a thermal beam are slowed in a Zeeman slower and trapped in a "blue" Magneto Optical Trap (MOT) operating on the ${ }^{1} \mathrm{~S}_{0^{-}}{ }^{1} \mathrm{P}_{1}$ resonance transition at $461 \mathrm{~nm}$. The temperature is further reduced by a second cooling stage in a "red" MOT operating on the ${ }^{1} \mathrm{~S}_{0^{-}}{ }^{3} \mathrm{P}_{1}$ intercombination transition at $689 \mathrm{~nm}$. This produces about $10^{6}$ atoms at a temperature of $0.6 \mu \mathrm{K}$. Since the force of gravity is comparable to the force produced by the red MOT on the atoms, the cloud of trapped atoms assumes a dish-like shape disk with a vertical size of $27 \mu \mathrm{m}$ and a radial size of $180 \mu \mathrm{m}$. The atoms are adiabatically loaded in an optical lattice in $300 \mu \mathrm{s}$. The lattice potential is generated by a singlemode frequency-doubled Nd:YVO 4 laser $\left(\lambda_{L}=532 \mathrm{~nm}\right)$

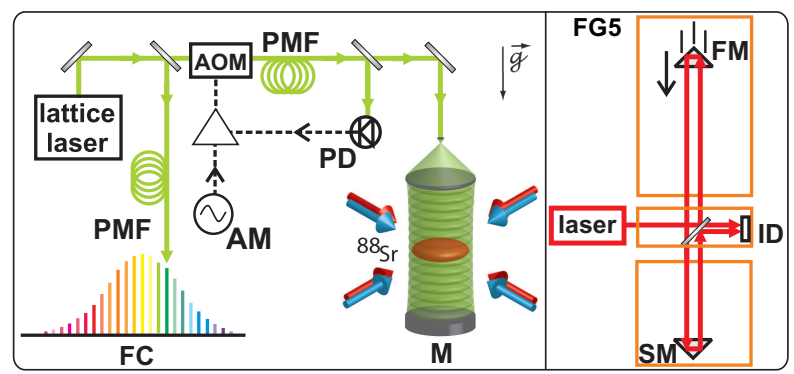

FIG. 1: Experimental setup for the measurement of gravity with ${ }^{88} \mathrm{Sr}$ atoms trapped in a vertical optical lattice and the comparison with a classical absolute gravimeter (FG5). M: lattice mirror; FC: frequency comb; FM: freely-falling mirror; SM: stationary mirror; ID: interference detector; AOM: acousto-optical modulator; PMF: polarization maintaining fiber; AM: RF generator for amplitude modulation of the laser producing the lattice; $\mathrm{PD}$ : photodiode. 


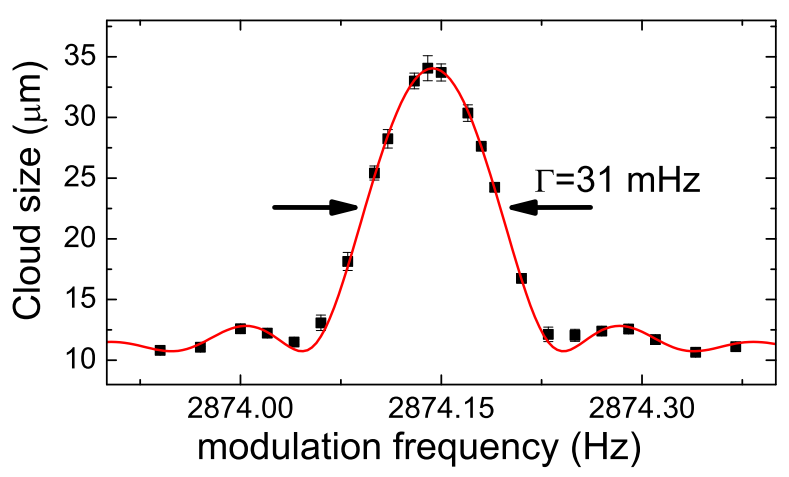

FIG. 2: Spectrum recorded by modulation of the lattice depth at the $5^{\text {th }}$ harmonic of the Bloch frequency for $10.4 \mathrm{~s}$ with a modulation depth of $7 \%$. The red line is a fit of experimental data with a sinc function.

delivering up to $1 \mathrm{~W}$ on the atoms with a beam waist of $557(7) \mu \mathrm{m}$. The beam is vertically aligned and retroreflected by a mirror. The atomic sample in the lattice has a vertical RMS size of about $14 \mu \mathrm{m}$ and a horizontal size of about $100 \mu \mathrm{m}$. The Bloch frequency is $\nu_{B} \simeq 574.3 \mathrm{~Hz}$ and the corresponding lattice photon recoil energy is $E_{R} \simeq 8 \mathrm{kHz} \times h$. In typical conditions the lattice depth ranges from 2.3 to $3 E_{R}$, while the energy gap $E_{G}$ at the recoil momentum $k_{L}$ is $E_{G} \simeq E_{R}$. The width of the first energy band in the lattice potential is about $0.5 \times E_{G}$. Landau-Zener tunneling is negligible in these conditions. The lattice depth is stabilized by a servo loop acting on the RF signal driving an acoustooptical modulator (AOM). The same AOM is also used to add an amplitude modulation to the lattice potential. The atomic cloud can be imaged either in situ or with usual time-of-flight technique using resonant absorption imaging on a CCD camera with a spatial resolution of $5 \mu \mathrm{m}$. The commercial lattice laser (Coherent V-5) employed in the measurement is not frequency stabilized and a precise calibration of its frequency is then required. To this purpose, part of the lattice laser light is sent to a home-built self-referenced Ti:Sa optical frequency comb. Due to residual frequency instabilities on the time scale of the Bloch frequency measurement, the uncertainty in the laser frequency is $\simeq 100 \mathrm{MHz}$. The correction for the index of refraction of the $\mathrm{Sr}$ cloud and the background gas in the vacuum chamber is negligible. The vertical alignment of the lattice was checked with a precision of $0.5 \mathrm{mrad}$, corresponding to a relative uncertainty of 10 ppb on $g$, by overlapping the downward laser beam with the reflection from the surface of water in a glass container inserted in the beam. A tiltmeter with a resolution of $1.7 \mu \mathrm{rad}$ attached to the optical table was employed to check the alignment stability during the measurements.

After loading ${ }^{88} \mathrm{Sr}$ atoms in the vertical lattice, the trap depth is modulated sinusoidally. When the modulation frequency matches an integer harmonic of the
Bloch frequency, atoms start to tunnel in neighbor lattice sites giving rise to a net increase in the spatial vertical atomic distribution which is observed in situ by resonant absorption imaging (see Fig2). The lifetime in the vertical lattice is about $20 \mathrm{~s}$. This allows us to apply amplitude modulation to the lattice for $\sim 10$ s resulting in an increased quality factor observed on the resonance at $\nu_{m}=5 \times \nu_{B}$. With a typical amplitude modulation depth of the order of $7 \%$ we estimate a tunneling rate $\mathcal{J}_{l} / \hbar=0.75$ [19]. The recording time for a whole resonance spectrum is about 1 hour and leads to a maximum resolution of $150 \mathrm{ppb}$ in $\nu_{B}$. The value of $g$ is given by $g=2 h \nu_{B} /\left(m_{\mathrm{Sr}} \lambda_{L}\right)$, where $m_{\mathrm{Sr}}$ is the mass of ${ }^{88} \mathrm{Sr}$ atoms and $h$ the Planck constant which are both known with a relative uncertainty of $\sim 5 \times 10^{-8}$.

An important contribution to systematic shifts in gravity measurements with trapped neutral atoms is due to the lattice light itself. Both the intensity and the wavevector of the lattice beam, which results from the interference of two counter-propagating Gaussian beams, yield space-dependent terms to the potential $U_{t o t}(z)=$ $U_{s}(z)+U_{l}(z) \cos (2 k(z) z)-m g z$, where $U_{s}(z)$ and $U_{t}(z)$ depend on the squared sum and on the product of the two beam field amplitudes, respectively. This additional dependence of the potential along the vertical direction gives rise to two correction terms in the typical Bloch formula $g=2 h \nu_{B} /\left(m_{\mathrm{Sr}} \lambda_{L}\right)+\Delta g_{U}+\Delta g_{k}$ given by the spatial derivative of the potential and the spatial derivative of the difference between the Gouy phase [22] for the two beams at the position of the atomic cloud $z_{a t}$. The shift introduced by these two extra terms is estimated by a precise determination of the geometry of the incoming and the reflected trapping beams and the position of the cloud with respect to the beam waist, with a relative uncertainty of $1 \%$. Moreover, an independent determination of the transverse beam size at $z_{a t}$ has been done by measuring the axial and radial atomic trap oscillation frequencies through parametric heating technique [23, 24]. For typical experimental parameters, the two terms are

\begin{tabular}{lcc}
\hline \hline Effect & Correction & Uncertainty \\
\hline \hline Lattice wavelength & 0 & 2 \\
Lattice beam vertical align. & 0 & 0.1 \\
Stark shift (beam geometry) & $14.3 \div 17.3$ & 0.4 \\
Experiment timing & 0 & 0.2 \\
Tides & $-1.4 \div 0.9$ & $<0.1$ \\
Height difference & 4.3 & 0.2 \\
Refraction index & 0 & $<0.01$ \\
Fundamental constants & 0 & 0.7 \\
\hline Systematics total & $17.2 \div 22.5$ & 2.2 \\
\hline \hline
\end{tabular}

TABLE I: Systematic corrections and their associated uncertainties $\left(\times 10^{-7}\right)$ for the gravity measurement with ${ }^{88} \mathrm{Sr}$ atoms in the amplitude modulated optical lattice. 


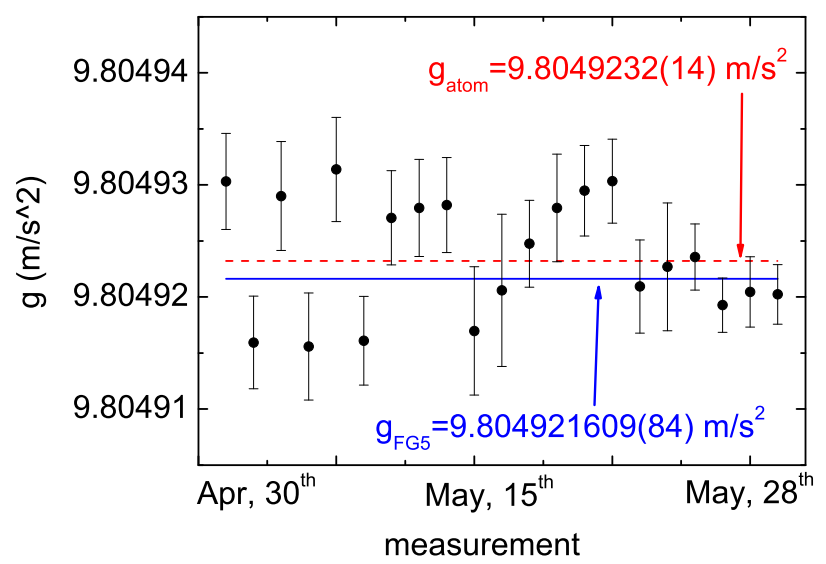

FIG. 3: (color online). Measurements of $g$ using the amplitude modulation technique. Each experimental point is corrected for the systematic effects presented in Tab. I] The red dashed line represents the weighted mean of the 21 measurements. The blue solid line is the value obtained with the classical absolute FG5 gravimeter.

$\Delta g_{U}=1.53(3) \times 10^{-5} \mathrm{~m} / \mathrm{s}^{2}$ and $\Delta g_{k}=1.0(2) \times 10^{-8} \mathrm{~m} / \mathrm{s}^{2}$.

Tidal effects were evaluated and removed from the raw data using the same algorithm and potential model used for the absolute gravimeter data processing. The peakto-peak effect of tides at our site is of the order of $2 \times$ $10^{-6} \mathrm{~m} / \mathrm{s}^{2}$. Since each measurement lasts about 1 hour the variation of $g$ during a single measurement due to tides is below $10^{-7} \mathrm{~m} / \mathrm{s}^{2}$ (i.e. below $10 \mathrm{ppb}$ ).

We checked also for a possible dependence from magnetic field gradients by performing a set of measurements applying a quadrupole magnetic field (from the MOT coils) up to $B=40$ gauss $/ \mathrm{cm}$. The effect on $\nu_{B}$ is smaller than the statistical error. All the other sources of systematic shift in the measurement we evaluated (spurious higher harmonics of amplitude modulation, Bloch-Siegert shift 25]) are far below the current accuracy level. Table II summarizes the systematic shifts for the gravity measurements with the amplitude modulation technique. The values of individual shifts depend on the experimental conditions; the quoted uncertainties are typical values.

The reference value for local gravitational acceleration $g$ was provided by an absolute gravimeter based on a Michelson interferometer with one arm including a freelyfalling corner-cube (FG5, Micro-g LaCoste). The measurement was performed in the same laboratory at a distance of $1.15 \mathrm{~m}$ from the atomic probe position. The difference in height of $14(5) \mathrm{cm}$ together with the estimated vertical gravity gradient value $g_{z z}=-3.09 \times 10^{-6} \mathrm{~S}^{-2}$ at the laboratory site was taken into account in the data analysis. The result is $g_{F G 5}=9.804921609(84) \mathrm{m} / \mathrm{s}^{2}$ [26].

Fig. 3 presents a set of 21 determinations of $g$ with ${ }^{88} \mathrm{Sr}$
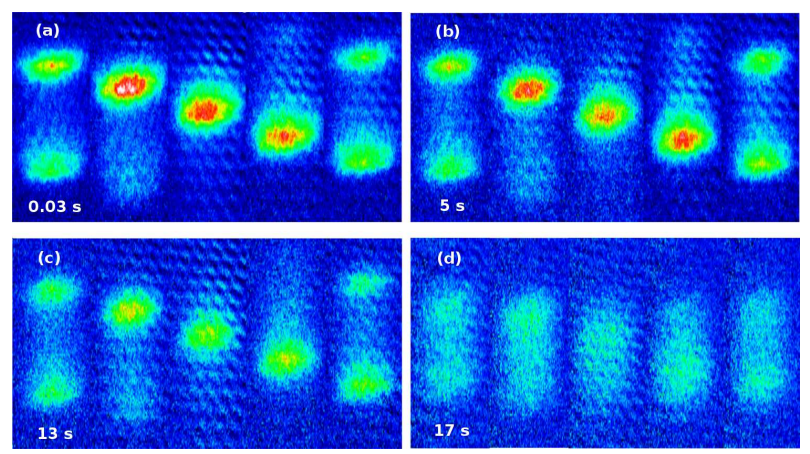

FIG. 4: (color online). Long-lived Bloch oscillations for $\mathrm{Sr}$ atoms in the vertical lattice under the influence of gravity. Each picture shows one Bloch cycle in successive time-of-flight absorption images giving the momentum distribution at the time of release from the lattice. Displayed are the first (a), the 2900th (b), the 7500th (c), and the 9800th (d) Bloch cycle.

atoms. The error bars are given by the quadrature sum of the statistical errors coming from the fit of the amplitude modulation resonance and the uncertainty on systematic corrections. The standard deviation is $\sigma=110 \mathrm{ppb}$ with a $\chi^{2}=30$. The resulting statistical uncertainty is $\sigma \times$ $\sqrt{\chi^{2} /(n-1)}=140 \mathrm{ppb}$. The weighted mean of our data is $g_{S r}=9.8049232(14) \mathrm{m} / \mathrm{s}^{2}$, in good agreement with the value obtained using the FG5 gravimeter.

With minor modifications of the experimental procedure, in this work we also determined $g$ by measuring the frequency of the Bloch oscillations of the atoms in the vertical optical lattice. Thanks to a better vacuum and taking advantage of the lattice modulation method to reduce the initial momentum distribution of the atoms in the lattice, we considerably improved the visibility and duration of the oscillations and, as a consequence, the frequency resolution compared with previous experiments [9]. After the transfer of ultracold atoms in the vertical optical lattice, an amplitude modulation burst with typical duration of 120 cycles at $\nu_{m} \simeq \nu_{B}$ is applied. The quantum phase of the atomic wavefunction induced by the amplitude modulation gives rise to an interference effect in time of flight image of the atomic cloud which results in an enhanced visibility of the Bloch oscillations peaks 27]. After the modulation has turned off, we let the atomic cloud evolve for a time T. Finally, we switch off the confinement within $5 \mu$ s to measure the momentum distribution taking an absorption picture on the CCD camera of the atoms in ballistic expansion. In order to optimize the visibility through this quantum interference effect we set the time-of-flight to $14 \mathrm{~ms}$. As shown in Fig. 4, we observe Bloch oscillations lasting up to $17 \mathrm{~s}$. From the the fit of the mean atomic momentum we can estimate the Bloch frequency with 170 ppb statistical uncertainty. In comparison with the determination of Bloch frequency obtained with the resonant amplitude modu- 
lation technique, however, we observed a considerably larger scattering in repeated measurements, mainly due to initial position instability of the atomic trap and also higher dependence on the timing of the experiment. The value for $g$ obtained with the Bloch oscillation technique is indeed $g_{\text {Bloch }}=9.80488(6) \mathrm{m} / \mathrm{s}^{2}$, which is consistent with the measurement discussed above but is affected by a larger uncertainty of $6 \mathrm{ppm}$.

It is important to notice that the amplitude modulation technique for gravity measurement allows further improvements of both accuracy and sensitivity. In fact our result is mainly limited by the lattice wavelength stability and by the Stark shift. The first effect could be lowered by using a tunable laser and locking it to an atomic line. For instance, if the wavelength is stabilized within $1 \mathrm{MHz}$, the uncertainty of this effect might be reduced by two orders of magnitude. The second main systematic effect could be reduced either by using a bluedetuned trapping laser [22] or by increasing the lattice beam waist. Also, the sensitivity could be increased by using higher harmonic amplitude modulation frequencies or by modulating for a longer time.

In conclusion, we performed an accurate measurement of gravitational acceleration using ultracold atoms trapped in a vertical optical lattice. The result is in agreement at $140 \mathrm{ppb}$ level with an independent determination obtained with a classical FG5 gravimeter. This result represents an improvement by an order of magnitude over previous results [9, 28] and is of interest as a test of the gravitational redshift [13]. Moreover we demonstrated the validity of the amplitude modulation technique [19] for the measurement of forces with high spatial resolution [10]. We also observed persistent Bloch oscillation up to $17 \mathrm{~s}$ which represents the longest coherence time observed to date. This result might also have important applications in precision measurements in conjunction with nondestructive cavity QED techniques to probe atomic momentum oscillations [29].

We thank ENI and INGV for the measurement with the FG5 gravimeter. We also thank M. Schioppo for his contribution in the early stage of the experiment, D. Sutyrin for help with absolute frequency measurements and R. Ballerini, M. De Pas, M. Giuntini, A. Hajeb, A. Montori for technical assistance. This work is supported by INFN and LENS (under contract RII3 CT 2003 506350).

* Also: ICTP, Trieste, I

† Present address: Institut für Angewandte Physik der Universität Bonn, Wegelerstrasse 8, 53115 Bonn, D

‡ Permanent address: Dipartimento di Fisica, Università di Bologna, Via Irnerio 46, 40126 Bologna, I

$\S$ Electronic address: Guglielmo.Tino@fi.infn.it

[1] A. D. Cronin, J. Schmiedmayer, and D. E. Pritchard, Rev. Mod. Phys. 81, 1051 (2009).
[2] A. Peters, C. K. Yeow, and S. Chu, Nature 400, 849 (1999).

[3] J. M. McGuirk, G. T. Foster, J. B. Fixler, M. J. Snadden, and M. A. Kasevich, Phys. Rev. A 65, 033608 (2002).

[4] J. B. Fixler, G. T. Foster, J. M. McGuirk, and M. Kasevich, Science 315, 74 (2007).

[5] G. Lamporesi, A. Bertoldi, L. Cacciapuoti, M. Prevedelli, and G. M. Tino, Phys. Rev. Lett. 100, 050801 (2008).

[6] S. Merlet, A. Kopaev, M. Diament, G. Geneves, A. Landragin, and F. Pereira Dos Santos, Metrologia 45, 265 (2008).

[7] M. D. Angelis, A. Bertoldi, L. Cacciapuoti, A. Giorgini, G. Lamporesi, M. Prevedelli, G. Saccorotti, F. Sorrentino, and G. M. Tino, Meas. Sci. Technol. 20, 022001 (2009).

[8] P. Clade, S. Guellati-Khelifa, C. Schwob, F. Nez, L. Julien, and F. Biraben, Europhys. Lett. 71, 730 (2005).

[9] G. Ferrari, N. Poli, F. Sorrentino, and G. M. Tino, Phys. Rev. Lett. 97, 060402 (2006).

[10] F. Sorrentino, A. Alberti, G. Ferrari, V. V. Ivanov, N. Poli, M. Schioppo, and G. M. Tino, Phys. Rev. A 79, 013409 (2009).

[11] H. Müller, S.-w. Chiow, S. Herrmann, S. Chu, and K.-Y. Chung, Phys. Rev. Lett. 100, 031101 (2008).

[12] G. Amelino-Camelia, C. Laemmerzahl, F. Mercati, and G. M. Tino, Phys. Rev. Lett. 103, 171302 (2009).

[13] H. Mueller, A. Peters, and S. Chu, Nature 463, 926 (2010).

[14] S. Fray, C. A. Diez, T. Hänsch, and M. Weitz, Phys. Rev. Lett. 93, 240404 (2004).

[15] S. Dimopoulos, P. W. Graham, J. M. Hogan, and M. A. Kasevich, Phys. Rev. D 78, 042003 (2008).

[16] G. M. Tino and F. Vetrano, Class. Quantum Grav. 24, 2167 (2007).

[17] S. Dimopoulos, P. W. Graham, J. M. Hogan, M. A. Kasevich, and S. Rajendran, Phys. Rev. D 78 (2008).

[18] G. M. Tino, L. Cacciapuoti, K. Bongs, C. Bord, P. Bouyer, H. Dittus, W. Ertmer, A. Görlitz, M. Inguscio, A. Landragin, et al., Nuclear Physics B (Proc. Suppl.) 166, 159 (2007).

[19] A. Alberti, G. Ferrari, V. V. Ivanov, M. L. Chiofalo, and G. M. Tino, New J. Phys. 12, 065037 (2010).

[20] P. Wolf, L. Blanchet, C. J. Borde, S. Reynaud, C. Salomon, and C. Cohen-Tannoudji, Nature 467, E1 (2010).

[21] H. Mueller, A. Peters, and S. Chu, Nature 467, E2 (2010).

[22] P. Cladé, E. de Mirandes, M. Cadoret, S. GuellatiKhélifa, C. Schwob, F. Nez, L. Julien, and F. Biraben, Phys. Rev. A 74, 052109 (2006).

[23] T. A. Savard, K. M. O'Hara, and J. E. Thomas, Phys. Rev. A 56, R1095 (1997).

[24] R. Jáuregui, N. Poli, G. Roati, and G. Modugno, Phys. Rev. A 64, 033403 (2001).

[25] F. Bloch and A. Siegert, Phys. Rev. 57, 522 (1940).

[26] M. De Angelis et al., to be published (2010).

[27] A. Alberti, V. V. Ivanov, G. M. Tino, and G. Ferrari, Nature Physics 5, 547 (2009).

[28] V. V. Ivanov, A. Alberti, M. Schioppo, G. Ferrari, M. Artoni, M. L. Chiofalo, and G. M. Tino, Phys. Rev. Lett. 100, 043602 (2008).

[29] B. M. Peden, D. Meiser, M. L. Chiofalo, and M. J. Holland, Phys. Rev. A 80, 043803 (2009). 